\title{
Dynamics of flux penetration and critical currents in type-II superconductors
}

\author{
J. Shumway* and S. Satpathy \\ Department of Physics, University of Missouri, Columbia, Missouri 65211
}

(Received 21 October 1996)

\begin{abstract}
We study the dynamics of flux penetration and vortex pinning in a type-II superconductor by numerical simulations with forces appropriate in the London regime. Straight vortex lines at zero temperature are studied in a slab geometry, and, unlike previous studies, full account is taken of the dynamical penetration of the flux lines across the surface under the application of an external magnetic field. Our simulation produces flux penetration in the sample beyond $H_{c 1}$ as expected, with the flux lines forming an Abrikosov lattice in the absence of the pinning centers. The pinning centers impede flux penetration into the bulk and the resulting critical current decreases with increasing vortex density roughly in agreement with the modified Bean's model.
\end{abstract} [S0163-1829(97)00425-6]

\section{INTRODUCTION}

It is well-known that pinning centers in type-II superconductors impede the penetration and motion of flux lines resulting in larger critical currents. ${ }^{1}$ Recently, a number of simulations have been performed in order to gain insight into the behavior of flux motion in the presence of pinning centers. However, most of these studies have been devoted to the melting and transport properties of the bulk flux lattice. ${ }^{2-4}$ Realistic simulations of how pinning centers affect the flux penetration and the distribution of the flux lines in the sample with a changing magnetic field have started only recently. ${ }^{5,6}$ In this paper, we report the results of our simulations in a two-dimensional slab geometry, using dynamics derived from the London free energy.

\section{METHOD OF SIMULATION}

The simulation of vortex motion was performed with classical noninertial (overdamped) dynamics taking into account all forces experienced by the vortices. The geometry being considered is an infinite slab with a thickness much larger than the London penetration depth in the $x$ direction, and the vortices are taken to be straight lines parallel to the external field in the $z$ direction. In the classical phenomenological approach, the vortices obey a diffusive equation of motion ${ }^{7}$

$$
\mathbf{F}-a \mathbf{v} \times \hat{\mathbf{z}}-\eta \mathbf{v}=0,
$$

where $\mathbf{F}$ is the total force acting on the vortex, $a$ is the magnitude of the Magnus force, $\mathbf{v}$ is the vortex velocity, $\hat{\mathbf{z}}$ is directed along the length of the vortex, and $\eta$ is the viscosity. In addition, a stochastic term can be added to the force to simulate the heat bath. The force on a vortex is given by

$$
\mathbf{F}=\mathbf{F}_{v v}+\mathbf{F}_{p}+\mathbf{F}_{s}+\mathbf{F}_{j},
$$

where $\mathbf{F}_{v v}$ is the force due to vortex-vortex interaction, $\mathbf{F}_{p}$ is the force due to the pinning centers, $\mathbf{F}_{s}$ is the force due to the surface of the sample, and $\mathbf{F}_{j}$ is the Lorentz force on the vortex due to the applied current (which is zero in the simulation). The repulsive vortex-vortex force is given by the derivative of the interaction energy $E_{12}=\left(\phi_{0} / 4 \pi\right) B\left(r_{12}\right)$ be- tween two vortices in the bulk. The surface force comes from image vortices, surface screening currents, and the external applied field. In the present simulation, the pinning centers are described by Gaussian potential wells, whose strength is reduced near the surface, as described below, to take into account the fact that the vortices near the surface are not fully formed.

The determination of forces acting on a flux lines near a surface has been solved by Brandt for the case of arbitrarily shaped flux lines in the London limit. ${ }^{8}$ Here we present a shorter derivation for the special case of straight vortex lines, with particular emphasis on calculating the Gibbs potential, which is needed when vortices are allowed to enter and leave the system. Spatial derivatives of the Gibbs potential will determine the forces acting on the vortices. Following DeGennes, ${ }^{9}$ we start from London equation,

$$
\mathbf{B}+\lambda_{L}^{2} \nabla \times \nabla \times \mathbf{B}=\phi_{0} \sum_{i} \delta^{2}\left(\mathbf{r}-\mathbf{r}_{i}\right) \hat{\mathbf{z}},
$$

where $\lambda_{L}$ is the London penetration depth and the source term is a summation over vortex cores. Using Maxwell's equations to express kinetic energy of the supercurrent in terms of the curl of the magnetic field, the total energy can be written as an integral over the magnetic field $\mathbf{B}$,

$$
E=\frac{1}{8 \pi} \int\left(\lambda_{L}^{2}|\nabla \times \mathbf{B}|^{2}+\mathbf{B}^{2}\right) d V,
$$

where the region of integration is the interior of the superconductor excluding the vortex cores. The magnetic field in Eq. (4) is a sum of the contribution of the applied field and the contribution from the vortices. The contribution from the applied field is localized near the surface,

$$
B(x)=H_{\mathrm{ext}} \exp \left(\frac{-x}{\lambda_{L}}\right),
$$

where $x$ is the distance from the surface, and $H_{\text {ext }}$ is the external field. The contribution from the vortices is a sum over all vortices

$$
\sum_{i} B_{v}\left(r_{i}\right)=\sum_{i} \frac{\phi_{0}}{2 \pi \lambda_{L}^{2}} K_{0}\left(\frac{r_{i}}{\lambda_{L}}\right),
$$


where $r_{i}$ is the distance from the core of the $i$ th vortex, $\phi_{0}=\hbar c /(2 e)$ is the flux quanta, and $K_{0}$ is a modified Bessel function. The method of images may be applied to the vortices to satisfy the boundary condition of continuity of the magnetic field at the surface. We simply subtract image terms (not shown) when performing the sum in Eq. (6) so that $\sum_{i} B_{v}=0$ at the surface.

After the integration in Eq. (4) is performed, the Gibbs potential takes the form

$$
\begin{aligned}
G \equiv & E-\int \frac{\mathbf{B} \cdot \mathbf{H}}{4 \pi} d A=\sum_{i} \tau_{i}\left(x_{i}\right)+\sum_{i<j} E_{v}\left(r_{i j}\right) \\
& -\frac{H}{4 \pi} \sum_{i} \phi_{i}\left(x_{i}\right)+E_{\text {pin }}+\text { const. }
\end{aligned}
$$

The summations are over all real vortices - the effects of image vortices are included in the definition of $\tau_{i}\left(x_{i}\right), E_{v}$, and $\phi_{i}\left(x_{i}\right)$, where $x_{i}$ represents the distance from vortex $i$ 's core to the surface. The terms are the reduced vortex line tensions $\sum_{i} \tau\left(x_{i}\right)$, the vortex-vortex interaction energy $E_{v}\left(r_{i j}\right)$, and the total magnetic flux in the superconductor due to vortices, $\Sigma_{i} \phi\left(x_{i}\right)$. An additional term, $E_{\text {pin }}$ has been added to introduce the effects of pinning sites. The line tension $\tau(x)$ for a vortex near the surface, defined as the energy per unit length of a vortex tube, is reduced due to interaction with the image vortex. We define $\tau(x)$ to be the energy terms that arise solely from interactions of a single vortex a distance $x$ from the surface with itself, its image, and the applied field. The resulting reduced line tension is a function of distance $x$ from the surface,

$$
\tau(x)=\tau_{\text {bulk }}\left[1-\frac{K_{0}(2 x)}{K_{0}(\xi)}\right]=\frac{\phi_{0}^{2}}{\left(4 \pi \lambda_{L}\right)^{2}} \ln \left(\frac{\lambda}{\xi}\right)\left[1-\frac{K_{0}(2 x)}{K_{0}(\xi)}\right]
$$

where $\xi$ is the vortex core radius, and it reduces to the standard expression for the line tension $\tau_{\text {bulk }}$ for vortices far from the surface. The core radius, bulk line tension, and critical field $H_{c 1}$ are related by the well-known relationships 9

$$
\frac{H_{c 1} \phi_{0}}{4 \pi}=\tau_{\text {bulk }}=\left(\frac{\phi_{0}}{4 \pi \lambda}\right)^{2} \ln \left(\frac{\lambda_{L}}{\xi}\right) .
$$

The pair vortex-vortex energy, entering in Eq. (7), depends on both the distance $r_{i j}$ between the vortices and, near a surface, on the distance $r_{i j}^{\prime}$ from one vortex to the image of the other. It has the form

$$
E_{v}\left(r_{i j}\right)=\frac{\phi_{0}}{4 \pi} B\left(r_{i j}\right)-\frac{\phi_{0}}{4 \pi} B\left(r_{i j}^{\prime}\right),
$$

where $B(r)$ is defined in Eq. (6). The reduced vortex flux $\phi(x)$ is the total flux in a vortex that has its core a distance $x$ from the surface, and is given by

$$
\phi(x)=\phi_{0}\left[1-\exp \left(-x / \lambda_{L}\right)\right] .
$$

This equation implies that vortices near the surface have less than a quanta of flux, and follows from fluxoid quantization ${ }^{10}$ by solving $\mathbf{B}+\lambda_{L}^{2} \nabla \times \nabla \times \mathbf{B}=\delta^{2}\left(\mathbf{r}-\mathbf{r}_{0}\right)$ with surface $(B=0)$ boundary conditions. This term may be interpreted ${ }^{9}$ as the force of the surface screening currents pushing the vortices inward, since the screening currents have this exponential dependence.
Pinning causes a decrease in the Gibbs potential. We describe this decrease in the potential by a Gaussian of strength $\gamma$ and width $\sigma$. We scale the strength of the pinning potential with the vortex line tension so that the pinning strength decreases for pinning sites acting on less formed vortices near the surface. Thus a vortex near a pinning site decreases the Gibbs potential by an amount

$$
\Delta G=-\tau_{i}\left(x_{v}\right) \gamma \frac{1}{\sqrt{2 \pi \sigma^{2}}} \exp \left[\frac{-\left(\mathbf{r}_{\mathbf{v}}-\mathbf{r}_{\mathbf{p}}\right)^{2}}{2 \sigma^{2}}\right],
$$

where subscripts $v$ and $p$ on coordinates refer to the vortex and pinning site, respectively. We take our pinning sites to have an energy depth of $\gamma=0.15$ and a width of $\sigma=0.08 \lambda_{L}^{2}$. In our simulations, the pinning centers were either placed on a grid or arranged randomly with a chosen value of pin density.

Our algorithm includes two types of dynamics, viz., the continuous motions of the vortices in the superconductor and the discrete addition and removal of vortices at the surface. The continuous motion of vortices is described by molecular dynamics (MD) governed by Eq. (1). Forces are calculated at each MD time step, which determines the vortex velocities through Eq. (1), and then the vortices are moved a distance $\mathbf{v} \delta t$. We use discrete addition of vortices to reduce the effect of a surface barrier present in the model. This barrier, originally suggested by Bean, ${ }^{11}$ occurs when the vortex line tension, Eq. (8), increases faster than the Gibbs energy decrease due to demagnetization, $-H_{\mathrm{ext}} \phi(x) / 4 \pi$, as the vortex moves inward. This occurs for small values of $x$ and is more prominent at low external fields, disappearing completely at high fields due to the minimum length scale $\xi$ of the London model. Bean proposed that this barrier can be bypassed if surface defects or geometric demagnetization effects are present. ${ }^{11}$ This physical effect is included in our model by allowing vortices to enter at distances up to one penetration length $\lambda_{L}$ from the surface, which is beyond the largest part of the barrier. This eliminates most, but not all, of the effect of the barrier. In our simulation, vortex addition is accomplished by attempting to add a vortex at points near the surface, and accepting the vortex if it would cause a decrease in the Gibbs potential. Removal of a vortex occurs naturally if its motion causes it to move outside the sample.

\section{RESULTS}

To test our methods, we first performed the simulations in the slab geometry but without any pinning centers present. The external magnetic field, always parallel to the slab surface in our simulation, was slowly increased from zero. As expected, the magnetic field lines do not penetrate the sample until the lower critical field $H_{c 1}$ is applied. As the external field became slightly greater than $H_{c 1}$, vortex lines began entering at the surface and spread quickly into the interior. The external field was increased at a rate slow enough that during the simulation process, the vortex density remains more-or-less uniform throughout the interior. The vortices then arrange themselves in an Abrikosov lattice, shown in Fig. 1. The lattice has many defects because it cannot uniformly compress as the vortex density increases, but the sixfold coordination is easily seen. Finite temperature may anneal some of the defects, but surface forces make 


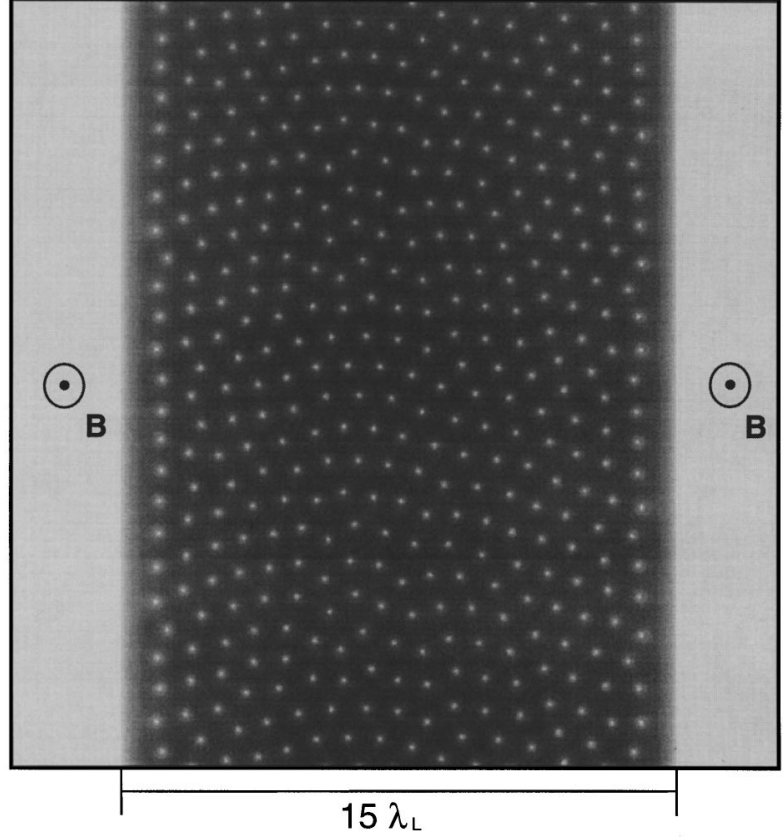

FIG. 1. A typical Abrikosov lattice seen in the simulations in the absence of pinning centers. The magnitude of the external field is $0.5 H_{c 1}$, directed out of the pages as indicated. The greyscale indicates magnetic-field intensity (lighter areas correspond to stronger fields). The light uniform regions on the sides indicate the applied external field. We have taken the magnus coefficient, defined in Eq. (1), to be $a=0.1$ in all simulations.

annealing more difficult in thin slabs. As predicted by Brandt $^{8}$ for the high vortex density limit $\left(d \ll \lambda_{L}\right.$, not reached in our simulations), we find that the separation of first lattice plane from the surface is slightly more than the internal lattice plane spacing.

The hysteresis curve for the case of no pinning is shown as a dashed line in Fig. 2, where it is compared to the bulk expression $^{12}$

$$
H=H_{c 1}+\frac{\phi_{0}}{16 \lambda_{L}^{2}} \sum_{i}\left\{2 K_{0}\left(\frac{r_{i}}{\lambda_{L}}\right)+\frac{r_{i}}{\lambda_{L}} K_{1}\left(\frac{r_{i}}{\lambda_{L}}\right)\right\},
$$

shown as a dotted line in the figure. The summation in this equation is over the sites of an Abrikosov lattice with density governed by the average internal magnetic field $B$. We find that vortex entry is delayed slightly past $H_{c 1}$, a consequence of the lingering surface barrier present in our model. Our hysteresis curve shows some departure from bulk results, also due in part to the presence of the surface barrier, which prevents vortices from entering. Another reason for departure from bulk results is that the defects in the simulated lattice keep the equilibrium vortex density lower. This would explain why the vortex density continues to be lower than the bulk theory even on ramp down. During ramp down, once the field has been lowered below about $1.5 H_{c 1}$, we find that surface hysteresis dominates over the energy cost of defects in this low density lattice, and the vortex density becomes higher than the bulk predictions. As the field is dropped to zero, a few vortices remain because the forces decay exponentially at large separations.

We then simulated the system with pinning present. Pinning impedes vortex flow, causing a gradient in the vortex

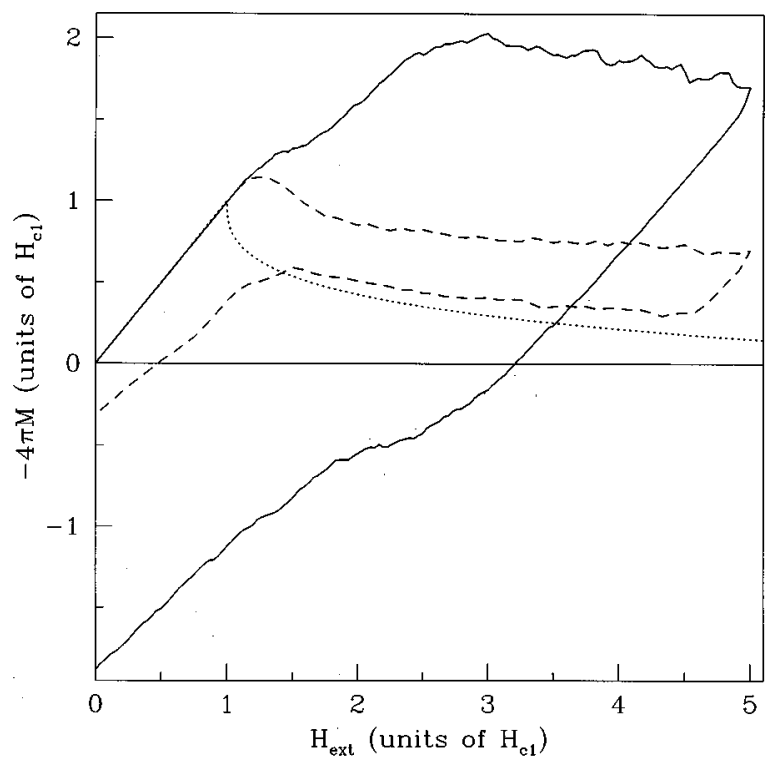

FIG. 2. Hysteresis curves for a superconductor with and without pinning. The dashed line is the computed hysteresis for no pinning, the solid line is with pinning present, and the dotted line is the analytical result for the infinite sample. For the increasing field, magnetic flux cannot fully penetrate, increasing the value of $-4 \pi M$. As the external field is decreased flux lines are trapped in the superconductor, causing a positive magnetization.

density to develop. For a choice of weak, dense pinning, the motion of all vortices is hindered, as opposed to the case of strong, sparse pinning, in which only a few individual vortices get pinned. The hysteresis curve for a pinned sample is shown as a solid line in Fig. 2. As the magnetic field is increased, flux entry is hindered, increasing the value of $-4 \pi M$ well above $H_{c 1}$. As vortices enter the sample, the magnetization levels off at around $-4 \pi M=2 H_{c 1}$. Physically, this plateau region corresponds to the first vortices reaching the center of the sample. When the external field is decreased, pinning hinders the escape of vortices from the sample, leading to a positive magnetization and a large hysteresis.

The magnetic profile at different values of $H_{\text {ext }}$ for flux entering a superconductor with pin density of ten sites per $\lambda_{L}^{2}$ is shown in Fig. 3. Bean's model ${ }^{13}$ postulates that the critical current, $J_{c}$, defined as the maximum slope of the magnetic profile, should be a constant. A fit of the Bean model is shown on the left side of Fig. 3 where we have taken $J_{c}=0.28 H_{c 1} / \lambda_{L}$. We have fit to the curve with the highest magnetic field because the model should be more accurate at higher vortex densities. There is approximate agreement, but the critical current seems to decrease with vortex density. A much better fit is shown on the right side of Fig. 3, where the form is taken from the modified Bean model of Kim, Hempstead, and Strnad. ${ }^{14}$ By studying the magnetization of $\mathrm{Nb}_{3} \mathrm{Sn}$ and $3 \mathrm{Nb}-\mathrm{Zr}$, they found an empirical relationship between $J_{c}$ and the local field $B$ given by $\alpha / J_{c}=B_{0}+B$. We find a good fit is given by taking $\alpha=4.5 H_{c 1}^{2} / \lambda_{L}$ and $B_{0}=12 H_{c 1}$. The simulations by Refs. 5 and 6 have also found agreement with this modified Bean model. 


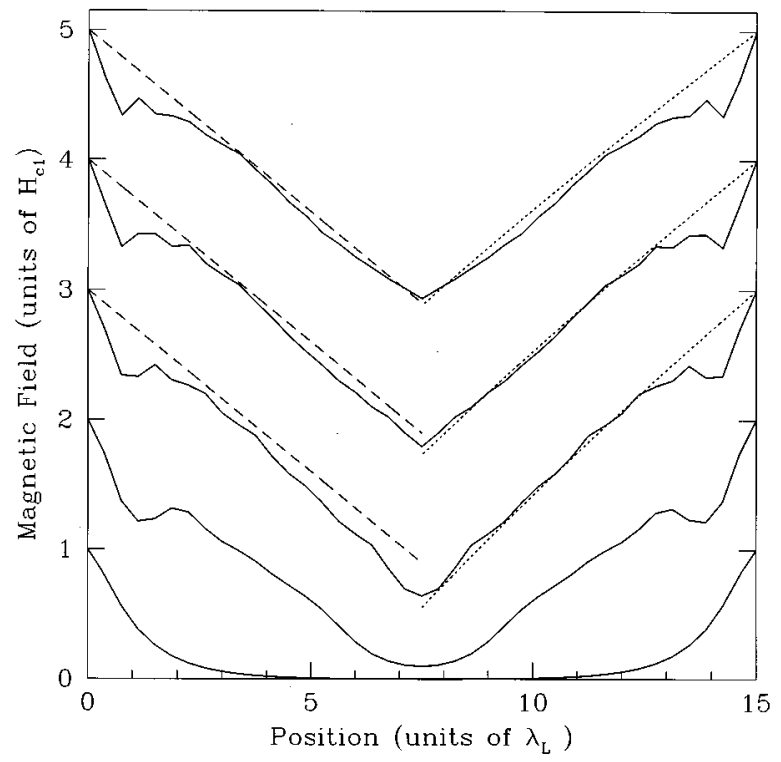

FIG. 3. Magnetic-flux profiles for a slab of thickness $15 \lambda_{L}$ and a pinning density of $10.0 \lambda_{L}^{-2}$ corresponding to different external magnetic fields $H_{\text {ext }}$. Abscissa denotes position along the thickness of the slab. Dashed lines (left-hand side) are a fit of the top level to the Bean model with $J_{c}=0.28 H_{c 1} / \lambda_{L}$. Dotted lines (right-hand side) are a fit to the modified Bean model with $\alpha=4.5 H_{c 1} / \lambda_{L}$ and $B_{0}=12 H_{c 1}$.

Further evidence for modified Bean model behavior can be seen from the hysteresis curve in Fig. 2. The plateau region should be constant for the Bean model, since it represents the integral of $B-H_{\text {ext }}$ across the sample, which is proportional to $J_{c}$. Under the modified Bean model, the value of this integral should be smaller at higher fields. This is clearly seen in the figure as the value of $-4 \pi M$ (solid line) decreases as the external field is increased from $3 H_{c 1}$ to $5 H_{c 1}$.

The magnitude of the critical current obtained from our simulation as a function of the pinning density is shown in Fig. 4. For comparison, we have evaluated $J_{c}$ at a common value of $B=3.0 H_{c 1}$, using our fitted modified Bean model parameters. We find that the critical current increases with pinning density, but then peaks at a pinning density of $15 \lambda_{L}^{-2}$, beyond which point our simulation indicates a decrease in the critical current. This optimal pinning density occurs just as the Gaussian potentials of pinning sites begin

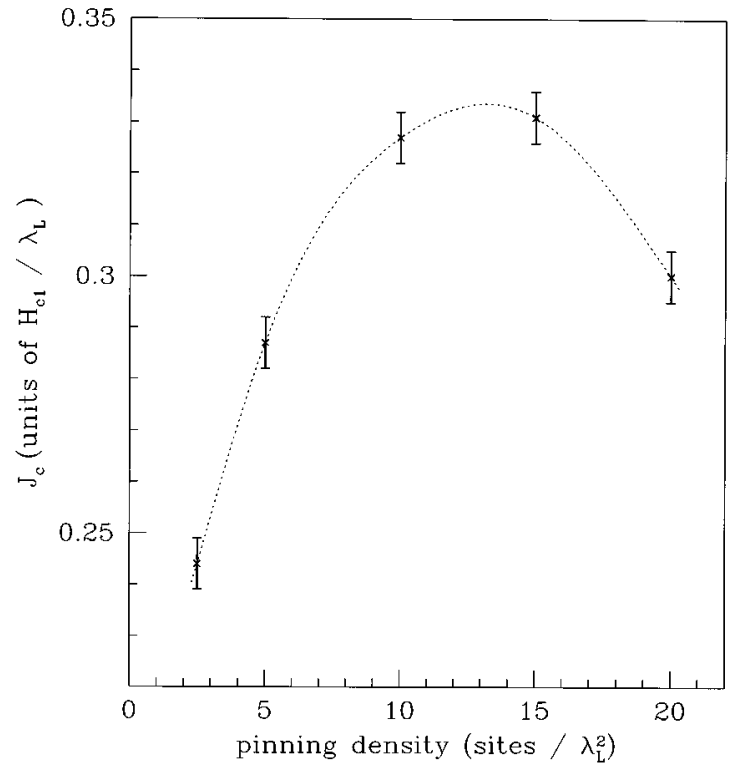

FIG. 4. Dependence of $J_{c}$ on the pinning density. Values of $J_{c}$ are compared at a common value $\left(B=3.0 H_{c 1}\right)$ of the average internal magnetic field. The dashed line guides the eye.

to overlap. The density at which the overlap begins depends on the form of the pinning potential, i.e., narrower pinning potentials would remain effective at higher pinning densities. Generalizing this result to other pinning potentials suggests that, for a given pinning interaction, the most efficient pinning is to have dense, but not overlapping, pinning sites.

In summary, we have simulated the dynamics of flux penetration into a superconductor, with an emphasis on studying the effects of pinning and flux penetration through the surface. By using realistic energies we have obtained reasonable behavior in the absence of pinning, in particular, we see flux expulsion below $H_{c 1}$ and the formation of an Abrikosov lattice above $H_{c 1}$. When pinning sites are present a gradient in flux distribution develops, which causes hysteresis and an increase in the critical current $J_{c}$ roughly following a modified Bean model. We find the most efficient pinning when pinning sites are dense but not overlapping.

\section{ACKNOWLEDGMENT}

We thank the Research Board of the University of Missouri for partial financial assistance.
*Present address: Department of Physics, University of Illinois, Urbana, IL 61801.

${ }^{1}$ For a review of flux vortex physics see, e.g., E. H. Brandt, Rep. Prog. Phys. 58, 1465 (1995); G. Blatter, M. V. Feigel'man, V. B. Geshkenbein, A. I. Larkin, and V. M. Vinokur, Rev. Mod. Phys. 66, 1125 (1994).

${ }^{2}$ A. Brass and H. J. Jensen, Phys. Rev. B 39, 9587 (1989).

${ }^{3}$ D. Reefman and H. B. Brom, Physica C 213, 229 (1993).

${ }^{4}$ J. Dong, J. Phys. Condens. Matter 5, 3359 (1993).

${ }^{5}$ R. A. Richardson, O. Pla, and F. Nori, Phys. Rev. Lett. 72, 1268 (1994).

${ }^{6}$ C. Reichhardt, C. J. Olson, J. Groth, S. Field, and F. Nori, Phys.
Rev. B 52, 10441 (1995).

${ }^{7}$ R. P. Huebener, Phys. Rep. 13c, 143 (1974).

${ }^{8}$ E. H. Brandt, J. Low Temp. Phys. 42, 557 (1981).

${ }^{9}$ P. G. De Gennes, Superconductivity of Metals and Alloys (Dover, New York, 1975).

${ }^{10}$ T. P. Orlando and K. A. Delin, Foundations of Applied Superconductivity (Addison-Wesley, New York, 1991).

${ }^{11}$ C. P. Bean and J. D. Livingstone, Phys. Rev. Lett. 12, 14 (1964).

${ }^{12}$ B. B. Goodman, Rep. Prog. Phys. 29, 445 (1966).

${ }^{13}$ C. P. Bean, Phys. Rev. Lett. 8, 250 (1962).

${ }^{14}$ Y. B. Kim, C. F. Hempstead, and A. R. Strnad, Phys. Rev. 129, 528 (1963). 